\section{Disease-modifying treatment in rheumatoid arthritis: treat early and do not forget to try to taper and stop}

We thank Professor Zeidler $^{1}$ for the interest in our study, in which we showed an increased chance and a shorter time to achieve disease-modifying antirheumatic drug (DMARD)-free sustained remission in patients with rheumatoid arthritis (RA) treated with present strategies (early start of methotrexate and treat-to-target approach) compared with treatment strategies that were applied 10 years ago. ${ }^{2}$ DMARD-free sustained remission in the study was defined as the sustained absence of synovitis (by physical examination) after discontinuation of DMARD therapy (including biologics and systemic or intra-articular corticosteroids) for the entire follow-up and at least for 1 year after DMARD withdrawal. ${ }^{3}$

Zeidler questioned if patients would also have achieved remission without DMARD treatment. Our study was done on an observational cohort of patients with RA (the Leiden Early Arthritis Clinic (EAC)); none of the patients studied were treated with placebo; therefore, we cannot formally answer this question.

Nonetheless, we do not believe that our results could be ascribed to a shift in inclusion of patients who would have had a spontaneous remission if they were left untreated, because of following reasons:

First, the inclusion process was similar throughout the recruitment years. Also, the achievement of DMARD-free sustained remission was uniformly assessed based on the medical records. We were thus able to compare the outcome of drug-free remission in the periods of changing initial treatment strategies in patients with RA.

Second, the diagnosis of RA in this large cohort was reassessed 1 year after inclusion. Patients who were not fulfilling 1987-American College of Radiology RA criteria and who were not prescribed any DMARDs the first year after inclusion, that is, who may have achieved spontaneous remission, were excluded from the study.

Third, the rate of remission in our study was higher in the patients diagnosed with RA in the latest decennium compared with those diagnosed in the 90 s, while spontaneous remission would occur regardless of treatment strategy and thus the rates of spontaneous remission would be similar through the years.

We agree with Zeidler that the severity of RA varies between patients, and the patients who achieve drug-free remission are generally the patients with milder disease characteristics (they are more frequently anti-citrullinated peptide antibody (ACPA) negative and have lower levels of inflammation at inclusion).
However, the shown difference in achieving DMARD-free remission between treatment strategies (inclusion periods) was independent of the swollen joint count, erythrocyte sedimentation rate/C reactive protein levels and autoantibody status at inclusion. Therefore, we concluded that improved treatment strategies resulted in an increased occurrence of DMARD-free sustained remission.

We believe that early suppression of disease activity can change the course of the disease and result in drug-free remission. We hope that our results encourage rheumatologists to consider reduction and withdrawal of DMARDs in patients with RA who were early treated according to current treatment strategies and have achieved sustained disease activity score (DAS) remission.

\section{S Ajeganova, ${ }^{1,2}$ AHM van der Helm-van Mil ${ }^{1}$}

${ }^{1}$ Department of Rheumatology, Leiden University Medical Center, Leiden, The Netherlands

${ }^{2}$ Department of Medicine Huddinge, Karolinska Institutet, Stockholm, Sweden

Correspondence to Dr S Ajeganova, Department of Medicine Huddinge, Karolinska Institutet, Stockholm 14186, Sweden; sofia.ajeganova@ki.se

Competing interests None declared.

Provenance and peer review Commissioned; internally peer reviewed.

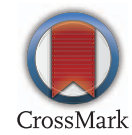

To cite Ajeganova S, van der Helm-van Mil AHM. Ann Rheum Dis 2017;76:e17.

Received 2 December 2016

Accepted 3 December 2016

Published Online First 23 December 2016

\section{Glinked}

http://dx.doi.org/10.1136/annrheumdis-2016-210887

Ann Rheum Dis 2017;76:e17. doi:10.1136/annrheumdis-2016-210892

REFERENCES

1 Zeidler $\mathrm{H}$. Drug-free sustained remission or spontaneous remission by natural history in rheumatoid arthritis? An unsolved question: comment on the article of Ajeganova et al. Ann Rheum Dis 2017;76:e16.

2 Ajeganova S, van Steenbergen HW, van Nies JA, et al. Disease-modifying antirheumatic drug-free sustained remission in rheumatoid arthritis: an increasingly achievable outcome with subsidence of disease symptoms. Ann Rheum Dis 2016;75:867-73

3 van der Woude D, Young A, Jayakumar K, et al. Prevalence of and predictive factors for sustained disease-modifying antirheumatic drug-free remission in rheumatoid arthritis: results from two large early arthritis cohorts. Arthitis Rheum 2009;60:2262-71. 